\title{
Biocompatibility Tests and Adhesion Improvements for Hydrogen-Free Amorphous Carbon for Blood-Contacting Medical Devices
}

\author{
Yuya Yamato, ${ }^{1 \dagger}$ Shunto Maegawa, ${ }^{1 \dagger}$ Terumitsu Hasebe, ${ }^{1,2{ }^{*}}$ \\ Kenta Bito, ${ }^{1}$ Tomohiro Matsumoto, ${ }^{1,2}$ Takahiko Mine, ${ }^{2}$ \\ Toshihiko Hayashi, ${ }^{2}$ Asushi Hotta, ${ }^{1}$ and Tetsuya Suzuki ${ }^{1}$ \\ ${ }^{1}$ Center for Science of Environment, Resource and Energy, Graduate School of Science and Technology, \\ Keio University, 3-14-1 Hiyoshi, Kohoku-ku, Yokohama, Kanagawa 223-8522, Japan \\ ${ }^{2}$ Department of Radiology, Tokai University Hachioji Hospital, Tokai University School of Medicine, \\ 1838 Ishikawa-machi, Hachioji, Tokyo 192-0032, Japan
}

(Received January 12, 2016; accepted May 9, 2017)

Keywords: amorphous carbon, biomaterial, adhesive properties, biocompatibility

The hydrogenated amorphous carbon $(\mathrm{a}-\mathrm{C}: \mathrm{H})$ film has been known as a coating material that imparts improved biocompatibility to base materials and has been used for many clinical applications. Recent studies have revealed that hydrogen-free amorphous carbon (H-free a-C) on stainless steel (SUS) has beneficial antibacterial properties, which allow avoidance of the risk of bacterial infection. In our study, to evaluate the biocompatibility of $\mathrm{H}$-free a-C itself for blood-contacting devices, we investigated not only the ability to grow bacteria but also platelet aggregation on the surfaces of $\mathrm{H}$-free a-C. Moreover, to apply $\mathrm{H}$-free a-C to polytetrafluoroethylene (PTFE), we evaluated the adhesive properties of $\mathrm{H}$-free a-C deposited after $\mathrm{Ar}$ or $\mathrm{O}_{2}$ plasma pre-treatment and fluorine-incorporated a-C:H (a-C:H:F) interlayer deposition. Antibacterial tests and antithrombogenic tests indicated that $\mathrm{H}$-free a-C coating reduced bacterial adhesion and platelet activation in comparison with a-C:H coating. The adhesive strength of plasma-treated Ar and the interlayer deposited PTFE was five times larger than those of untreated PTFE from film adhesion tests. These results indicated that the pre-treated PTFE coated with $\mathrm{H}$-free $\mathrm{a}-\mathrm{C}$ is a promising candidate biomaterial for medical devices.

\section{Introduction}

Hydrogenated amorphous carbon (a-C:H), which consists of $\mathrm{sp}^{3}$ and $\mathrm{sp}^{2}$ carbon bonds that include hydrogen in their structure, has been attracting attention as a biomaterial, showing a number of good properties such as extreme hardness, low friction coefficient, high wear resistance, and good biocompatibility. ${ }^{(1)}$ Actually, studies of new biomaterials proposed to enhance the antibacterial and antithrombogenic properties of a-C:H, ${ }^{(2-4)}$ as well as the addition of metal atoms, have been promising in terms of improving the antibacterial properties of a-C:H..$^{(5,6)}$ However, these materials have not reached the stage of practical applications to medical devices owing to the outflows of metal ions and damage to living bodies. Recently, Zhou et al. have reported that

\footnotetext{
*Corresponding author: e-mail: hasebe@tokai-u.jp

${ }^{\dagger}$ These authors contributed equally to this work.

http://dx.doi.org/10.18494/SAM.2017.1573
}

ISSN 0914-4935 C MYU K.K. 
amorphous carbon films applied to SUS with a reduced hydrogen content inhibited the adhesion and colonization of bacteria. ${ }^{(7)}$ Thus, it is expected that hydrogen-free amorphous carbon (H-free a-C) materials would be more practical as coatings for medical devices.

Polytetrafluoroethylene (PTFE) is one of the most useful biomaterials used for biliary tubes and covering materials for biliary stents as well as for prosthetic vascular grafts. However, bacterial infection through microbial adhesion and thrombus formation by the adsorption of blood components on the PTFE surface are potentially serious obstacles. ${ }^{(8-10)}$ Therefore, H-free a-C coated on PTFE is one of the best ways to address such problems.

Generally, amorphous carbon exhibits poor adhesion with other base materials used for medical implants, which causes film cracking. Some reports have revealed that plasma pretreatment improves the adhesion of amorphous carbon. ${ }^{(11-13)}$ Thus, appropriate deposition processing, including pretreatment, is required when amorphous carbon films are used for coatings.

In our study, we measured the antibacterial and antithrombogenic properties to verify the biocompatibility of the $\mathrm{H}$-free a-C itself. Then, to further improve the adhesive strength between PTFE and $\mathrm{H}$-free a-C, we deposited $\mathrm{H}$-free a-C onto a PTFE substrate using not only the plasma deposition method after $\mathrm{Ar}$ or $\mathrm{O}_{2}$ gas plasma treatment, but also the introduction of an interlayer of fluorine-incorporated a-C:H (a-C:H:F) during pretreatment.

\section{Materials and Methods}

\subsection{Biocompatibility tests}

\subsubsection{Sample preparation: Deposition of amorphous carbon films}

Two types of carbon film, a-C:H and $\mathrm{H}-$ free a-C, were deposited on PTFE or Si substrates. The a-C:H was deposited by radio frequency (RF) plasma-enhanced chemical vapor deposition (CVD) from $\mathrm{C}_{2} \mathrm{H}_{2}$. The $\mathrm{RF}(13.56 \mathrm{MHz})$ power and total pressure were fixed at $200 \mathrm{~W}$ and 13.3 $\mathrm{Pa}$, respectively. The $\mathrm{H}$-free a-C was deposited by filtered arc deposition (FAD). A graphite target was used as the cathode material. Deposition was carried out with an arc voltage and an arc current of $100 \mathrm{~V}$ and $40 \mathrm{~A}$, respectively. The total pressure was fixed at $2 \times 10^{-3} \mathrm{~Pa}$. The total thickness of the carbon films was about $50 \mathrm{~nm}$.

\subsubsection{Characterizations of amorphous carbon films}

For characterization of amorphous carbon films, we deposited a-C:H and $\mathrm{H}$-free a-C films on Si substrates under the conditions described in Sect. 2.1.1 to exclude the effects of substrates' surface morphology and roughness. Structural information of the carbon films was obtained using an argon laser Raman spectrometer (NRS-2100, Jasco, Tokyo, Japan) and Fourier transform infrared spectroscopy (FT-IR, BRUKER ALPHA-T). Raman spectroscopy was performed at room temperature using the $514.5 \mathrm{~nm}$ line from the Ar laser, and the laser power was kept constant at $1 \mathrm{~mW}$ at the sample surface. Spectra were recorded from 1100 to $1700 \mathrm{~cm}^{-1}$ and fitted using two Gaussian peaks after subtracting the background to determine the peak positions. FT-IR was employed to evaluate the $\mathrm{C}-\mathrm{H}$ bonding state in the carbon films. The transmission spectra were recorded in the range of $1000-4000 \mathrm{~cm}^{-1}$.

The surface chemical composition was measured by high-resolution elastic recoil detection analysis (HR-ERDA) using $480 \mathrm{keV} \mathrm{N}^{+}$ions, directed at the sample surface at an incident angle of $70^{\circ}$ 
relative to the sample normal. The energy of the hydrogen ions, recoiled out of the samples, was detected using the magnetic field deflecting energy analyzer at a position such that the scattering angle was $30^{\circ}$. The exposure dose was determined by measuring the electric current irradiated in the pendulum, which caused the pendulum to vibrate within the beam path. The detection limit of each element was approximately 2 at.\% of carbon and oxygen and 1 at.\% of hydrogen.

The wettability and surface free energy of carbon films, such as $\mathrm{H}$-free a-C and a-C:H, were evaluated by measuring the static contact angles between a droplet $(2 \mathrm{ml})$ of distilled water and diiodomethane, and the sample surface at room temperature. The water contact angles were taken from the averages of ten samples. Contact angle measurements were conducted by the sessile drop method with a DM 500 (Kyowa Interface Science Co., Ltd.).

\subsubsection{Antibacterial tests}

Escherichia coli (E. coli) was selected as an indicator for experimental bacteria. We prepared untreated PTFE, PTFE coated with a-C:H, and PTFE coated with $\mathrm{H}$-free a-C. Each substrate was $50 \times 50 \mathrm{~mm}^{2}$. Three samples were evaluated by the colony-counting method. All the samples were sterilized by autoclaving at $121{ }^{\circ} \mathrm{C}$ for $20 \mathrm{~min}$. A solution of bacteria in sterile phosphatebuffered saline (PBS) with a concentration of $5 \times 10^{5} \mathrm{CFU} / \mathrm{ml}$ was applied in droplets to the surface of the samples equally and evenly with a bacteria solution at a density of $0.5 \mathrm{ml}$ per sample. Then, all the samples were incubated at $36{ }^{\circ} \mathrm{C}$ and $>90 \% \mathrm{RH}$ for $24 \mathrm{~h}$, as called for by the JIS $\mathrm{Z}$ 2801 standard. After the incubation, the samples were rinsed with PBS and then in soybean-casein digest broth with lecithin and polysorbate (SCDLP) for several seconds to wash out the bacteria that had become attached to the sample surface. Subsequently, serial dilutions of the extracted solution were spread on agar plates and incubated at $36{ }^{\circ} \mathrm{C}$ for two nights. Colonies were counted visually, and the numbers of CFU per sample were determined.

\subsubsection{Antithrombogenic tests}

Human whole blood $(90 \mathrm{ml})$ was collected from healthy volunteers who had not taken any medication for at least 10 days and mixed with $10 \mathrm{ml}$ of acid-citrate-dextrose (AC-D). Following this, the blood was centrifuged at $350 \times g$ for $15 \mathrm{~min}$ to separate the blood corpuscles, and thus the resulting platelet-rich plasma (PRP) was prepared. Subsequently, a portion of the PRP was centrifuged at $1000 \times g$ for $10 \mathrm{~min}$ to obtain the platelet-poor plasma (PPP). The density of platelets in PRP was adjusted to $3.0 \times 10^{5} / \mathrm{ml}$ by dilution with PPP. Sample disks (surface area of $10 \times 10 \mathrm{~mm}^{2} ; n=4$ disks per sample), a control (polycarbonate), a Si substrate coated with a-C:H, and a Si substrate coated with $\mathrm{H}$-free a-C were washed with phosphate-buffered saline (PBS) at $\mathrm{pH}$ 7.4 and then incubated in 24-well plates containing $1 \mathrm{ml}$ of adjusted PRP at $37{ }^{\circ} \mathrm{C}$ for $60 \mathrm{~min}$ in an atmosphere containing $5.0 \% \mathrm{CO}_{2}$ gas. Thereafter, the supernatant was discarded, and the samples were washed with PBS. The adherent platelets were then fixed for $60 \mathrm{~min}$ at room temperature in $0.8 \mathrm{ml}$ of freshly prepared $1.0 \%$ glutaraldehyde. After fixation, the samples were washed and dehydrated in a graded ethanol series $(20,40,60,80,100$, and $100 \%$ for 10 min each), as described previously. ${ }^{(14)}$ The entirely dried materials were examined using differential interference contrast (DIC) microscopy (Nikon ECLIPSE L150, Tokyo, Japan). Adhering platelets were manually counted using photographs, and the number per unit area $\left(12800 \mu \mathrm{m}^{2}\right)$ was calculated. The platelet morphology was classified in accordance with the following five grades as described by Goodman 
et al. and Allen et al:: (1) round or discoid, (2) dendritic or early pseudopodial, (3) spread-dendritic or intermediate pseudopodial, (4) spreading or late pseudopodial, and (5) fully spread. ${ }^{(15,16)}$

\subsection{Film adhesion evaluation}

\subsubsection{Sample preparation: Plasma treatment and deposition of amorphous carbon films}

The PTFE substrates of about $0.1 \mathrm{~mm}$ thickness were treated with $\mathrm{Ar}$ or $\mathrm{O}_{2}$ plasma using RF-CVD. The RF (13.56 MHz) power and total pressure were fixed at $200 \mathrm{~W}$ and $13.3 \mathrm{~Pa}$, respectively. The PTFE substrates were modified in Ar or $\mathrm{O}_{2}$ plasma for 30-300 s. After the plasma treatment on PTFE substrates, interlayers of a-C:H:F on the substrates were deposited by RF-CVD. The a-C:H:F films were deposited on the substrates using a mixture of acetylene $\left(\mathrm{C}_{2} \mathrm{H}_{2}\right)$ and hexafluoroethane $\left(\mathrm{C}_{2} \mathrm{~F}_{6}\right)$ under the same conditions as those used for plasma treatment. The a-C:H:F films were deposited at a partial pressure of $\mathrm{C}_{2} \mathrm{H}_{2}$ at $20 \%$ and $\mathrm{C}_{2} \mathrm{~F}_{6}$ at $80 \%$. Two types of carbon film, a-C:H and $\mathrm{H}$-free a-C, were deposited on the pretreated PTFE substrates. The a-C:H films and $\mathrm{H}$-free a-C films were deposited under the conditions described in Sect. 2.1.1.

\subsubsection{Characterizations of the plasma treated PTFE and the interlayer}

After the plasma treatment and introduction of the a-C:H:F interlayer on the PTFE substrates, surface chemical compositions were measured by X-ray photoelectron spectroscopy (XPS, JPS9010TR, JEOL Ltd., Tokyo Japan). The X-ray source employed here was Al Ka, and the spectral regions used for the measurement were $\mathrm{Cls}$, O1s, and F1s.

\subsubsection{Adhesive tests}

A T-peel test (ISO11339:2003) was conducted to evaluate adhesive properties. To determine peel strength, we prepared untreated PTFE directly coated with H-free a-C (no pretreatment), $\mathrm{O}_{2}$ - or Ar-plasma-treated PTFE directly coated with $\mathrm{H}$-free a-C $\left[\mathrm{O}_{2}\right.$ (no interlayer) and $\mathrm{Ar}$ (no interlayer), respectively]. and $\mathrm{O}_{2}$ - or Ar-plasma-treated PTFE coated with $\mathrm{H}$-free a-C $\left[\mathrm{O}_{2}\right.$ (no interlayer) and $\mathrm{Ar}$ (no interlayer), respectively]. Each sample was bonded using epoxy resin (Devcon S-31, ITW Industry Ltd.). The samples were then pressed at $5 \mathrm{MPa}$ at room temperature for $300 \mathrm{~s}$ to remove any trapped air at the adhesive interface. The pressed samples were then placed in an oven set at $50{ }^{\circ} \mathrm{C}$ to accelerate the hardening of the epoxy adhesive. After hardening, the samples were cut into portions with areas of $100 \times 25 \mathrm{~mm}^{2}$, as per the standard procedure provided by International Organization for Standardization (ISO). (The length was slightly modified to fit the chamber of our RF-CVD system.) The T-peel test was carried out using a tensile tester (AG-50NIS MS of Shimadzu Corporation). Two free edges of one sample were grasped by two clamps of the tensile tester. The lower clamp was settled at the same position and the upper clamp was free to move upward at a predefined velocity of $10 \mathrm{~mm} / \mathrm{min}$ provided by ISO.

\subsection{Statistical analysis}

SPSS software version 22 was used for statistical analysis. The results were expressed as the mean values with the corresponding standard deviation. Comparisons of the peel strength of each sample in plasma treatment time were analyzed using one-way analysis of variance (ANOVA) with 
post-hoc Dunnett's test. For statistical analysis, quantitative variables were compared via $t$-test. The differences were considered to be statistically significant when the value of $p$ was less than 0.05 .

\section{Results and Discussion}

\subsection{Characterizations of amorphous carbon films}

Figure 1 shows structural information on the samples after the deposition of carbon films by Raman spectroscopy. There were two peaks: the D-peak $\left(1350 \mathrm{~cm}^{-1}\right)$ and the G-peak $\left(1530 \mathrm{~cm}^{-1}\right)$, which indicate a representative diamond-like carbon film, in the Raman spectrum of both a-C:H and $\mathrm{H}$-free a-C.

Figure 2 shows the chemical structure of carbon films observed by FT-IR. According to the study of Zhou et al., as the peak meant $-\mathrm{CH}_{3}\left(2870 \mathrm{~cm}^{-1}\right)$ and $-\mathrm{CH}_{2}\left(2925\right.$ and $\left.2950 \mathrm{~cm}^{-1}\right)$, this is direct evidence of the existence of hydrogen. ${ }^{(7)}$ The FT-IR spectrum of $\mathrm{H}$-free a-C was quite flat in the range of $1000-4000 \mathrm{~cm}^{-1}$. On the other hand, there was a peak at $2910 \mathrm{~cm}^{-1}$ in the FT-IR spectrum of a-C:H. We speculated that the peak at $2910 \mathrm{~cm}^{-1}$ was a complex formed of peaks of $-\mathrm{CH}_{2}$ and $-\mathrm{CH}_{3}$. Therefore, as the peak was observed for the a-C:H in our study, the presence of $\mathrm{C}-\mathrm{H}$ bonds on the a-C:H was confirmed, and because no peak was observed for the $\mathrm{H}$-free a-C in our study, the suppression of $\mathrm{C}-\mathrm{H}$ bonds on the $\mathrm{H}$-free a-C was confirmed.

Except for a contaminated layer, the surface chemical composition of the a-C:H of our study consisted of 60.1 at.\% carbon, 39.9 at.\% hydrogen, and 0.0 at.\% oxygen on high-resolution elastic recoil detection analysis (HR-ERDA). On the other hand, $\mathrm{H}$-free a-C of our study consisted of 100 at.\% carbon on HR-ERDA. Such results directly indicated that there was no hydrogen on the $\mathrm{H}-\mathrm{free}$ a-C in our study.

Table 1 shows the water contact angle and surface free energy of carbon films. The denotation " $\theta_{w}$ " represents the contact angle $\left({ }^{\circ}\right) . \gamma_{\mathrm{s}}, \gamma_{\mathrm{s}}{ }^{p}$, and $\gamma_{\mathrm{s}}{ }^{d}$ represent the surface free energy and its polar and dispersive components with reference to the carbon films, respectively. The value of $\theta_{w}$ on $\mathrm{H}$-free a-C was $69.0^{\circ}$ and on a-C:H, it was $65.9^{\circ}$. The water contact angle of amorphous carbon films depends on surface roughness and surface chemical composition. As two carbon films, $\mathrm{H}$-free a- $\mathrm{C}$ and a-C:H are very smooth [i.e., when deposited on a Si substrate, the surface roughness $(R a)$ was less than $1 \mathrm{~nm}$ ], and the water contact angle has no decisive effect on the surface roughness

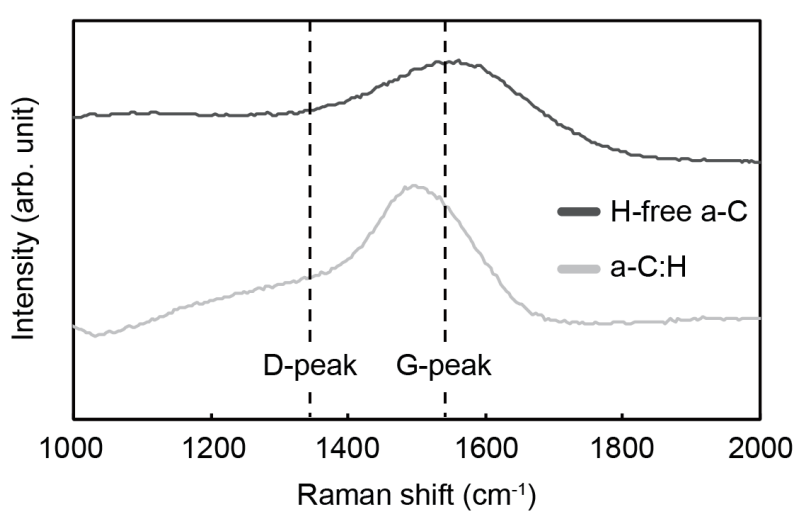

Fig. 1. Raman spectra of two carbon films.

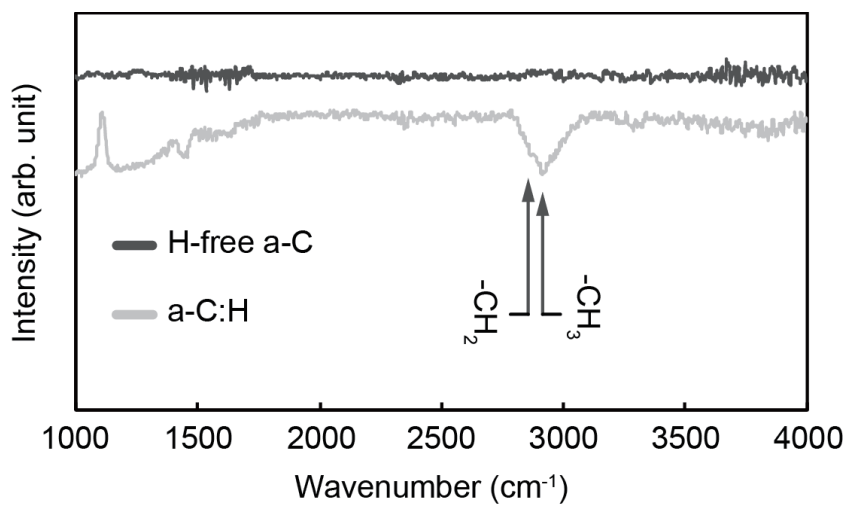

Fig. 2. FT-IR spectra of two carbon films. 
Table 1

Water contact angle and surface free energy of two carbon films.

\begin{tabular}{lcccc}
\hline & $\theta_{w}$ & $\gamma_{\mathrm{s}}{ }^{d}\left(\mathrm{~mJ} / \mathrm{m}^{2}\right)$ & $\gamma_{\mathrm{s}}{ }^{p}\left(\mathrm{~mJ} / \mathrm{m}^{2}\right)$ & $\gamma_{\mathrm{s}}\left(\mathrm{mJ} / \mathrm{m}^{2}\right)$ \\
\hline a-C:H & $65.9 \pm 0.7$ & $41.4 \pm 1.1$ & $8.8 \pm 0.5$ & $50.2 \pm 1.2$ \\
H-free a-C & $69.0 \pm 1.0$ & $44.7 \pm 1.3$ & $5.3 \pm 0.6$ & $50.0 \pm 1.4$ \\
\hline
\end{tabular}

in this study. The surface chemical composition difference between a-C:H and $\mathrm{H}$-free a-C was exhibited by the existence (or lack thereof) of an C-H bond in the film. According to Ma et al., $\mathrm{C}-\mathrm{H}$ bonds are characteristic of "oil", which is hydrophobic in nature. ${ }^{(17)}$ However, as reported previously, the water contact angle of $\mathrm{H}$-free a-C is greater than $80^{\circ} .{ }^{(18)}$ Therefore, the result of wettability depends on deposition condition or film formation equipment, and the water contact angle of our $\mathrm{H}$-free a-C was $69.0^{\circ}$. In addition, the water contact angle of our a-C:H film was $65.9^{\circ}$, and the result was similar to that measured by our group. ${ }^{(19,20)}$ The polar components $\left(\gamma_{\mathrm{s}}{ }^{p}\right)$ of $\mathrm{H}$-free a-C were lower than those of a-C:H, while the dispersion components $\left(\gamma_{\mathrm{s}}{ }^{d}\right)$ of $\mathrm{H}$-free a-C were higher than those of a-C:H, with the total surface free energy $\left(\gamma_{s}\right)$ on $\mathrm{H}$-free a-C and a-C:H having the same value. These results are due to differences in surface chemical composition, especially with regard to $\mathrm{C}-\mathrm{H}$ bonds. $\mathrm{C}-\mathrm{H}$ bonds are known as polar bonds, in contrast to $\mathrm{C}-\mathrm{C}$ bonds. Therefore, since $\mathrm{H}$-free a-C exhibited fewer $\mathrm{C}-\mathrm{H}$ bonds and more $\mathrm{C}-\mathrm{C}$ bonds than a-C:H, the polar components of $\mathrm{H}$-free $\mathrm{a}-\mathrm{C}$ were lower than those of a-C:H, and the dispersion components of $\mathrm{H}$-free a-C were higher than those of a-C:H.

\subsection{Biocompatibility tests}

Figure 3 shows the average quantities of E. coli adhered to untreated PTFE, the PTFE coated with a-C:H, and the PTFE coated with $\mathrm{H}$-free a-C. Figure 4 shows the representative images of colony adhesion to the surface of each sample. The average quantities of $E$. coli on the a-C:H and $\mathrm{H}$-free a-C were each significantly lower than that on the PTFE substrate. Moreover, the average quantity of $E$. coli on the $\mathrm{H}$-free a-C was approximately one-twentieth of that on the a-C:H.

Figure 5 shows the number of platelets per unit area adhering to the surfaces of the control and substrates coated with a-C:H and $\mathrm{H}$-free a-C after $60 \mathrm{~min}$ of incubation with PRP. The number of platelets adhering to a-C:H and $\mathrm{H}$-free a-C was significantly lower when compared to that on the control. In addition, the number of platelets adhering to $\mathrm{H}$-free a-C was approximately one-third of that on a-C:H.

Figure 6 shows representative images of platelet adhesion to the surfaces of the control and substrates coated with a-C:H and $\mathrm{H}-$ free a-C by scanning electron microscopy (SEM). Platelets adhering to the control had become spread out and flattened owing to their activation, whereas on $\mathrm{H}$-free a-C, a few platelets were attached; the morphology of such platelets was spherical and they had not become activated. The surface of a-C:H exhibited both platelets that had become flattened and spread out, as well as those that had remained spherical.

Figure 7 shows the number of adherent platelets per unit area according to morphological grade. Platelets adhering to the control surface were frequently grade 5 , whereas those on $\mathrm{H}$-free a-C were mostly grades 1 and 2 . The surface of a-C:H exhibited the presence of platelets of all grades.

On the basis of our results, $\mathrm{H}$-free a-C was found to be a material with excellent antibacterial and antithrombogenic properties. We consider that the surface characterizations of carbon films 


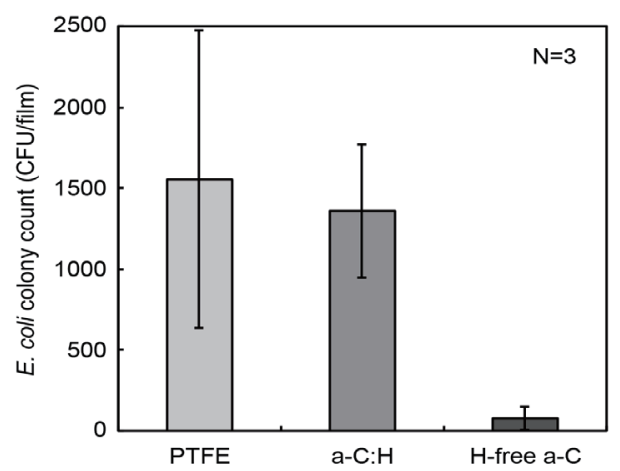

Fig. 3. Average quantities of $E$. coli adhered to untreated PTFE, a-C:H, and $\mathrm{H}$-free a-C.

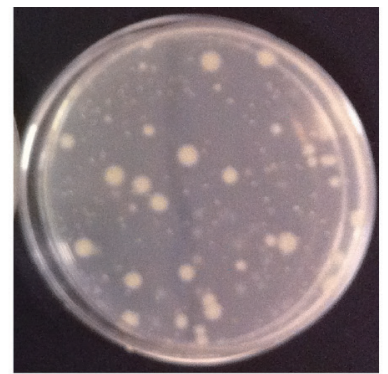

(a)

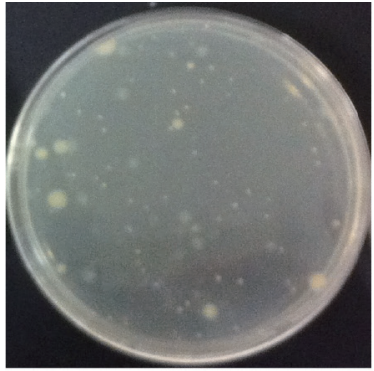

(b)

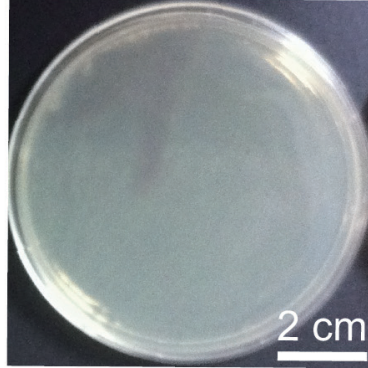

(c)

Fig. 4. (Color online) Representative images of E. coli adhered to (a) untreated PTFE, (b) a-C:H, and (c) H-free a-C.

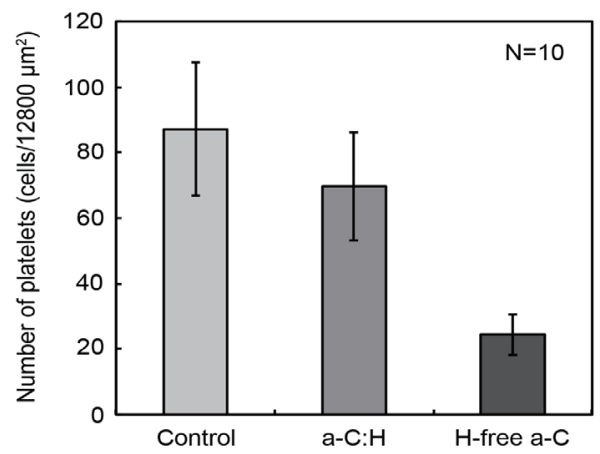

Fig. 5. Number of platelets adhered to control (polycarbonate), a-C:H, and H-free a-C.

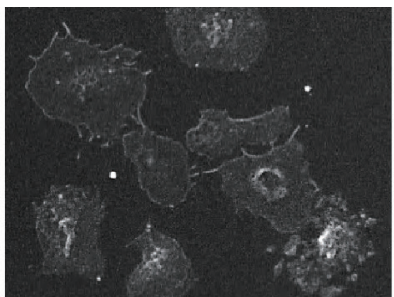

(a)

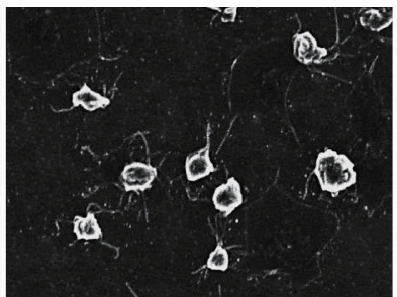

(b)

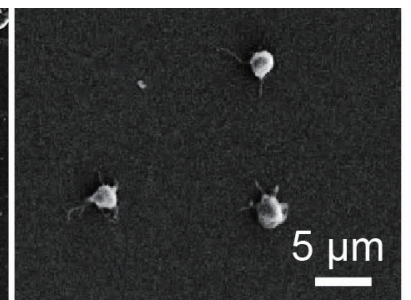

(c)

Fig. 6. Representative SEM images of platelets adhered to (a) control (polycarbonate), (b) a-C:H, and (c) H-free a-C. 


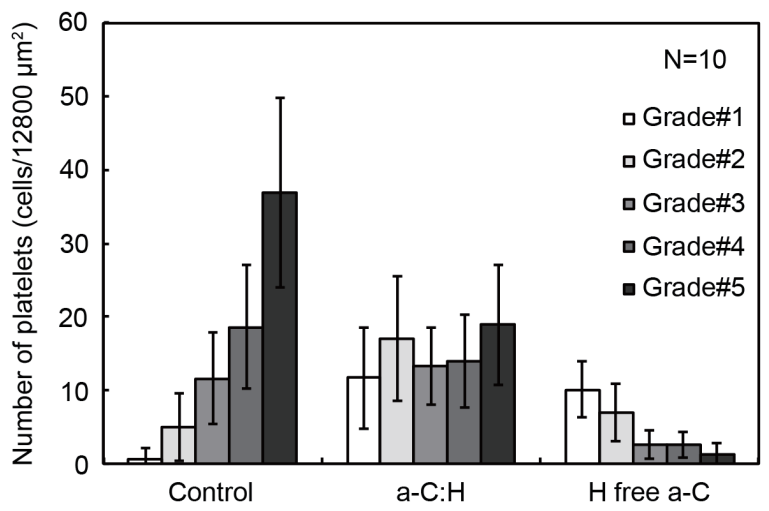

Fig. 7. Number of platelets adhered to control (polycarbonate), a-C:H, and $\mathrm{H}$-free a-C according to morphological grade.

affect cell activity. According to Zhou et al., the antibacterial properties of $\mathrm{H}$-free a-C may result from its chemical inertness because of weakened chemical interaction in the bacteria adhesion process. ${ }^{(7)}$ Moreover, it is said that the interactions between bacteria and its adhesion surface include electrostatic and van der Waals forces. ${ }^{(21)}$ In the components of surface free energy, the polar component $\left(\gamma_{s}^{p}\right)$ of $\mathrm{H}$-free a-C was lower than that of a-C:H, while the dispersion component $\left(\gamma_{s}{ }^{d}\right)$ of $\mathrm{H}$-free a-C was higher than that of a-C:H (Table 1). Therefore, there is a possibility that difference in the polar components of a-C:H and $\mathrm{H}$-free a-C affects the antibacterial properties.

On the other hand, there have been several reports that platelet adhesion to amorphous carbon films is related to wettability. For example, more hydrophilic surfaces promote a greater affinity for platelet attachment and spreading than hydrophobic amorphous carbon. ${ }^{(22)}$ In addition, the amorphous carbon family exhibits improved antithrombogenic properties with relatively hydrophobic surfaces compared with conventional amorphous carbon. ${ }^{(23-27)}$ Therefore, we considered, as one potential factor, the possibility that hydrophobic $\mathrm{H}$-free a-C suppressed the adhesion and activation of platelets. Moreover, surface free energy is also one factor in biomaterial-induced thrombosis. ${ }^{(28)}$ In particular, the low surface free energy can result in the reduced adhesion of platelets. ${ }^{(19)}$ In this study, however, there was no difference between the surface free energies $\left(\gamma_{\mathrm{s}}\right)$ of $\mathrm{H}$-free a-C and a- $\mathrm{C}: \mathrm{H}$; therefore, the surface free energies of $\mathrm{H}$-free a-C and a-C:H cannot be related to suppress adhesion or activation of platelets. According to Park et al., the platelet adhesion of films increases owing to the increase in the polar components. ${ }^{(29)}$ Hence, H-free a-C suppressed thrombus formation owing to fewer polar components. Furthermore, Ma et al. proposed that since albumin adsorption increases with reduction in the hydrogen content of the a- $\mathrm{C}: \mathrm{H}$, the higher the ratio of albumin/fibrinogen adsorption on the film is, the more the antithrombogenic properties of the film improved, ${ }^{(17)}$ which may support our discussion.

However, the adhesive behavior of bacteria and platelets on a material is more complex and further investigation is needed to clarify which factors affect the complex behavior.

\subsection{Characterizations of the plasma-treated PTFE and the interlayer}

Figure 8 shows the surface composition for each plasma treatment time on the PTFE substrates, as measured via XPS. The surface chemical composition of untreated PTFE consisted of about 


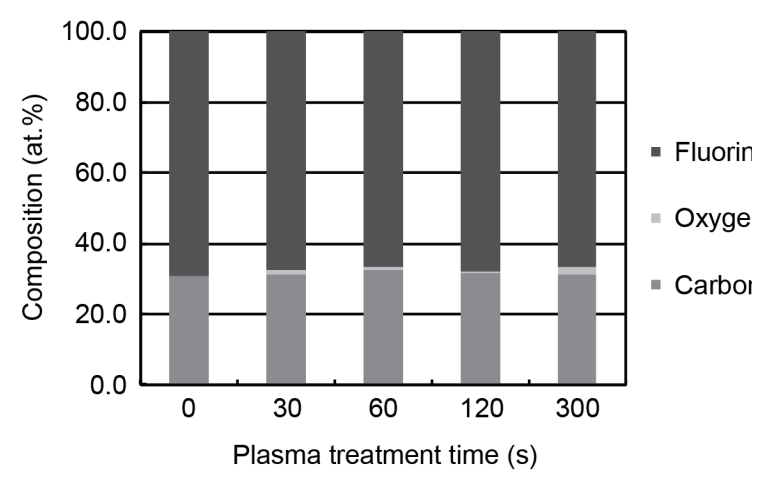

(a)

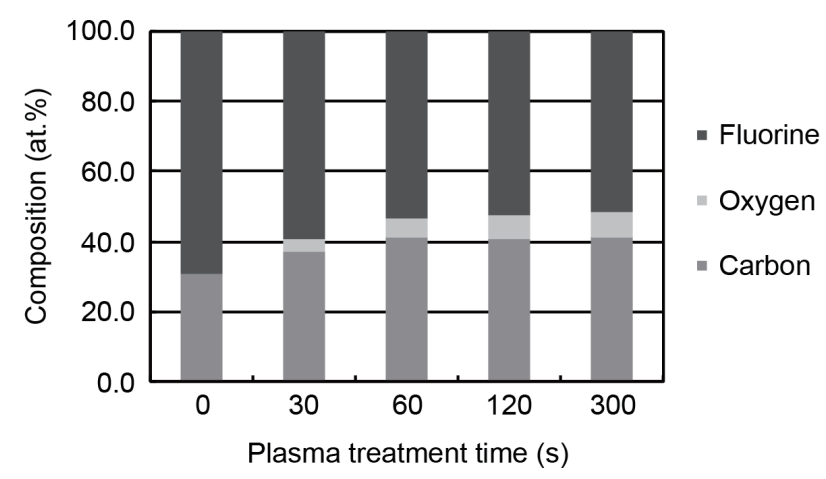

(b)

Fig. 8. Chemical composition of PTFE surface after (a) $\mathrm{O}_{2}$ plasma and (b) Ar plasma treatments by XPS.

30.9 at.\% carbon and 69.1 at.\% fluorine, and these figures were close to the theoretical values. Approximately 0.2 at.\% oxygen was also detected, but the signal was too small for reliable deconvolution. The surface chemical composition of the a-C:H:F on PTFE consisted of about 43.1 at. $\%$ carbon, 1.8 at. $\%$ oxygen and 55.1 at.\% fluorine.

In $\mathrm{O}_{2}$ plasma treatment, the C1s spectrum was similar to that of untreated PTFE [Fig. 9(a)]. Moreover, there was no significant difference between the F/C ratio for untreated PTFE and that of the $\mathrm{O}_{2}$-plasma-treated PTFE. The study results of Ozeki and Hirakuri also showed no functional group was formed on the PTFE surface during $\mathrm{O}_{2}$ plasma treatment, ${ }^{(11)}$ which was consistent with the results of our study. In Ar plasma treatment, the concentration of fluorine decreased from 69.1 to 51.4 at.\%, and the concentration of oxygen increased to 7.3 at.\% after $300 \mathrm{~s}$ of exposure [Fig. 8(b)]. The F/C ratio decreased immediately from 2.2 to 1.3 at.\% with increasing the plasma treatment time. Moreover, the peaks of $\mathrm{C}-\mathrm{O}$ and $\mathrm{C}=\mathrm{O}$ bonds in $\mathrm{C} 1$ s spectra increased and those of CF2 bonds decreased as Ar plasma treatment time increased [Fig. 9(b)]. From the results, it can be stated that Ar plasma treatment caused the desorption of fluorine and the introduction of oxygen on PTFE. The introduction of oxygen on PTFE surfaces during nonoxygen plasma treatment is a common phenomenon. ${ }^{(30,31)}$ Free radicals that are created on a PTFE surface during treatment in Ar plasma can react with atmospheric oxygen. ${ }^{(31)}$

\subsection{Adhesive tests}

The peel strengths of $\mathrm{O}_{2}$ (interlayer), $\mathrm{O}_{2}$ (no interlayer), and no pretreatment are shown in Fig. 10(a), and those of $\mathrm{Ar}$ (interlayer), $\mathrm{Ar}$ (no interlayer), and no pretreatment are shown in Fig. 10(b). The peel strengths of all the plasma-treated samples improved in comparison with the no pretreatment case [Figs. 10(a) and 10(b)].

The peel strengths of $\mathrm{O}_{2}$ (interlayer) and $\mathrm{O}_{2}$ (no interlayer) were independent of plasma treatment time. In comparing $\mathrm{O}_{2}$ (interlayer) and $\mathrm{O}_{2}$ (no interlayer), the peel strength of $\mathrm{O}_{2}$ (interlayer) was lower than that of $\mathrm{O}_{2}$ (no interlayer). With Ar plasma treatment, there was no difference in the peel strength of $\mathrm{Ar}$ (no interlayer) given different plasma treatment times. On the other hand, the peel strength of Ar (interlayer) increased proportionally with the plasma treatment time. When 


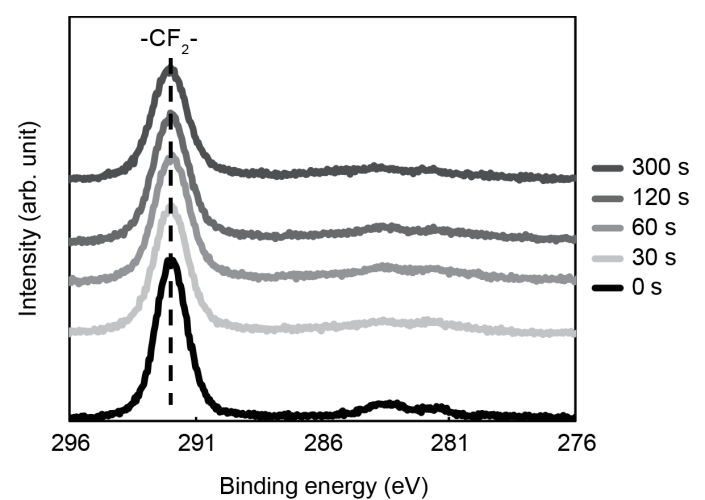

(a)

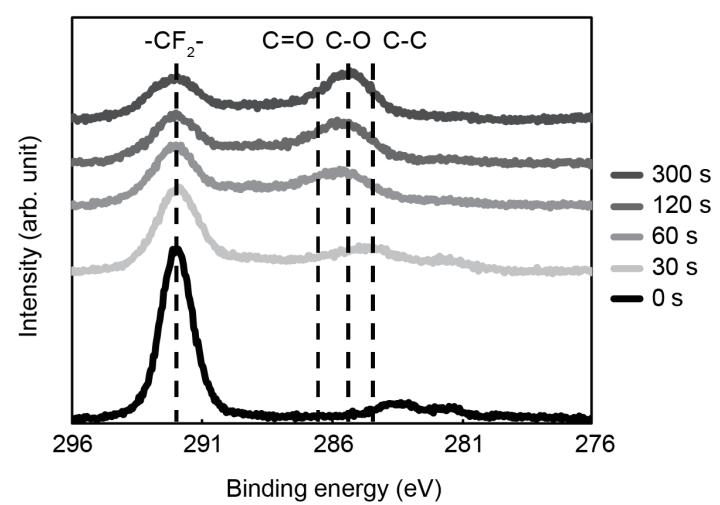

(b)

Fig. 9. C1s spectrum of PTFE surface after (a) $\mathrm{O}_{2}$ plasma and (b) Ar plasma treatments by XPS.

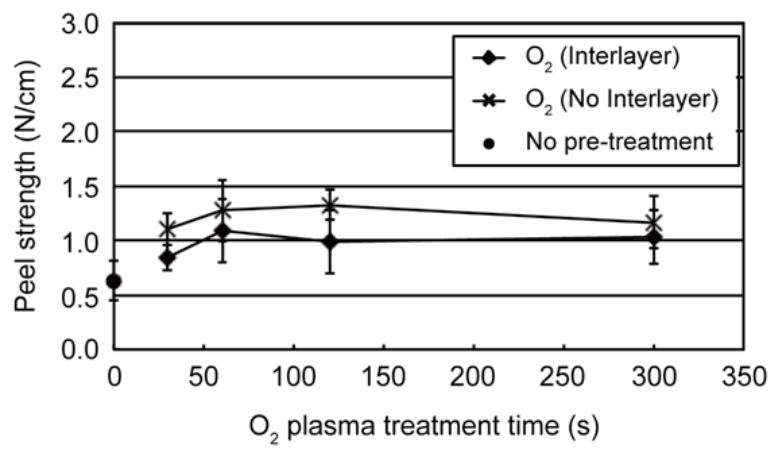

(a)

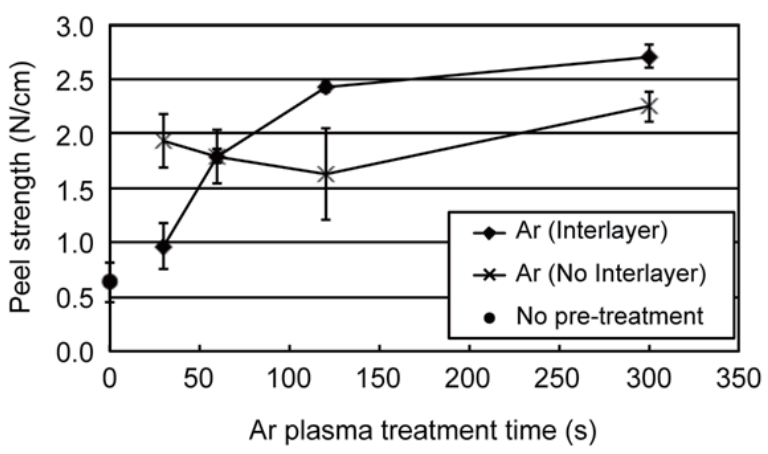

(b)

Fig. 10. Peel strength between $\mathrm{H}$-free a-C and PTFE with (a) $\mathrm{O}_{2}$ plasma treatments and (b) Ar plasma treatment via T-peel test.

$\operatorname{Ar}$ (interlayer) and Ar (no interlayer) are compared, the peel strength of Ar (interlayer) can be seen to be lower that of $\mathrm{Ar}$ (no interlayer) at a treatment time of $30 \mathrm{~s}$. However, the peel strength of $\mathrm{Ar}$ (interlayer) became higher than that of Ar (no interlayer) when plasma treatment took place during periods lasting over $60 \mathrm{~s}$.

The results indicated that the peel strength in cases involving PTFE and $\mathrm{H}$-free a-C improved with plasma treatment. This can be explained by the removal of the weak boundary layer (WBL). Since the problem of adhesive properties on PTFE was caused by WBLs such as foreign matter, impurities, additives, and low-molecular-weight substances, ${ }^{(32)}$ the increase in adhesive strength was attributed to the cleaning of the PTFE surface by $\mathrm{O}_{2}$ or Ar plasma treatment. There was no change in peel strength with plasma treatment over time involving the $\mathrm{O}_{2}$ (interlayer), $\mathrm{O}_{2}$ (no interlayer) or $\mathrm{Ar}$ (no interlayer) surfaces. On the other hand, the peel strength of Ar (interlayer) increased with plasma treatment time. These results could be related to the chemical composition of the PTFE surfaces. On the basis of Fig. 8(a), since it can be seen that the surface chemical composition of the $\mathrm{O}_{2}$-plasma-treated PTFE did not change, the peel strength of both $\mathrm{O}_{2}$ (interlayer) and $\mathrm{O}_{2}$ (no interlayer) did not improve, regardless of plasma treatment time. Looking at Fig. 8(b), 
it can be seen that the desorption of fluorine occurred with Ar plasma treatment, and the surface chemical composition became similar to that of the a-C:H:F interlayer. Consequently, the peel strength of $\mathrm{Ar}$ (interlayer) increased in proportion to plasma treatment time, whereas the peel strength of Ar (no interlayer) did not change with plasma treatment time. The peel strength of $\mathrm{O}_{2}$ (interlayer) was lower than that of $\mathrm{O}_{2}$ (no interlayer). The peel strength of $\mathrm{Ar}$ (interlayer) was also lower than that of Ar (no interlayer) with a treatment time of $30 \mathrm{~s}$. The $300 \mathrm{~s}$ Ar-plasmatreated PTFE coated with the interlayer proved to have the strongest adhesive properties among the samples used in the T-peel test.

\section{Conclusions}

The bacterial adhesion tests indicated that the $\mathrm{H}$-free a-C dramatically reduced bacterial adhesion in comparison with the untreated PTFE and a-C:H. Furthermore, the numbers of adhesive platelets and activated platelets were the lowest on the $\mathrm{H}$-free a-C surface, indicating that the surface was the most antithrombogenic among the samples. The surface of PTFE was improved in terms of adhesive properties via Ar plasma treatment and the introduction of the interlayer of a-C:H:F. These results indicated that the pretreated PTFE coated with $\mathrm{H}$-free a-C is a promising candidate biomaterial for medical devices.

\section{References}

1 P. Yang, N. Huang, Y. X. Leng, J. Y. Chen, R. K. Y. Fu, S. C. H. Kwok, Y. Leng, and P. K. Chu: Biomaterials 24 (2003) 2821.

2 H. J. Steffen, J. Schmidt, and A. Gonzalez-Elipe: Surf. Interface Anal. 29 (2000) 386.

3 J. Y. Chen, L. P. Wang, K. Y. Fu, N. Huang, Y. Leng, Y. X. Leng, P. Yang, J. Wang, G. J. Wan, H. Sun, X. B. Tian, and P. K. Chu: Surf. Coat. Technol. 156 (2002) 289.

4 T. Hasebe, T. Ishimaru, A. Kamijo, Y. Yoshimoto, T. Yoshimura, S. Yohena, H. Kodama, A. Hotta, K. Takahashi, and T. Suzuki: Diam. Relat. Mater. 16 (2007) 1343.

5 H. G. Kim, S. H. Ahn, J. G. Kim, S. J. Park, and K. R. Lee: Thin Solid Films 482 (2005) 299.

6 F. R. Marciano, L. F. Bonetti, L. V. Santos, N. S. Da-Silva, E. J. Corat, and V. J. Trava-Airoldi: Diam. Relat. Mater. 18 (2009) 1010.

7 H. Zhou, L. Xu, A. Ogino, and M. Nagatsu: Diamond Relat. Mater. 17 (2008) 1416.

8 A. Hatzidakis, M. Krokidis, K. Kalbakis, J. Romanos, I. Petrakis, and N. Gourtsoyiannis: Cardiovasc. Inter. Rad. 30 (2007) 950.

9 W. C. Johnson and K. K. Lee: J. Vasc. Surg. 32 (2000) 268.

10 J. Barrio, C. Ripoll, R. Banares, A. Echenagusia, M. V. Catalina, F. Camunez, G. Simo, and L. Santos: Eur. J. Radiol. 55 (2005) 120.

11 K. Ozeki and K. K. Hirakuri: Appl. Surf. Sci. 254 (2008) 1614.

12 Y. B. Guo and F. C. N. Hong: Diamond Relat. Mater. 12 (2003) 946.

13 K. Baba and R. Hatada: Thin Solid Films 506 (2006) 55.

14 R. D. Frank, H. Dresbach, H. Thelen, and H. G. Sieberth: J. Biomed. Mater. Res. 52 (2000) 374.

15 S. L. Goodman, T. G. Grasel, S. L. Cooper, and R. M. Albrecht: J. Biomed. Mater. Res. 23 (1989) 105.

16 R. D. Allen, L. R. Zacharski, S. T. Widirstky, R. Rosenstein, L. M. Zaitlin, and D. R. Burgess: J. Cell Biol. 83 (1979) 126.

17 W. J. Ma, A. J. Ruys, R. S. Mason, P. J. Martin, A. Bendavid, Z. W. Liu, M. Ionescu, and H. Zreiqat: Biomaterials 28 (2007) 1620.

18 C. Meunier, P. Alers, L. Marot, J. Stauffer, N. Randall, and S. Mikhailov: Surf. Coat. Technol. 200 (2005) 1976.

19 T. Hasebe, S. Yohena, A. Kamijo, Y. Okazaki, A. Hotta, K. Takahashi, and T. Suzuki: J. Biomed. Mater. Res. A 83 A (2007) 1192.

20 S. Nagashima, T. Hasebe, A. Kamijo, Y. Yoshimoto, A. Hotta, H. Morita, H. Terada, M. Tanaka, K. Takahashi, and T. Suzuki: Diamond Relat. Mater. 19 (2010) 861. 
21 Y. L. Ong, A. Razatos, G. Georgiou, and M. M. Sharma: Langmuir 15 (1999) 2719.

22 M. I. Jones, I. R. McColl, D. M. Grant, K. G. Parker, and T. L. Parker: Diamond Relat. Mater. 8 (1999) 457.

23 T. Saito, T. Hasebe, S. Yohena, Y. Matsuoka, A. Kamijo, K. Takahashi, and T. Suzuki: Diamond Relat. Mater. 14 (2005) 1116.

24 T. Hasebe, A. Shimada, T. Suzuki, Y. Matsuoka, T. Saito, S. Yohena, A. Kamijo, N. Shiraga, M. Higuchi, K. Kimura, H. Yoshimura, and S. Kuribayashi: J. Biomed. Mater. Res. A 76A (2006) 86.

25 T. Hasebe, S. Nagashima, A. Kamijo, T. Yoshimura, T. Ishimaru, Y. Yoshimoto, S. Yohena, H. Kodama, A. Hotta, K. Takahashi, and T. Suzuki: Thin Solid Films 516 (2007) 299.

26 T. Hasebe, A. Hotta, H. Kodama, A. Kamijo, K. Takahashi, and T. Suzuki: New Diamond Front. C. Technol. 17 (2007) 263.

27 T. I. T. Okpalugo, A. A. Ogwu, P. D. Maguire, and J. A. D. McLaughlin: Biomaterials 25 (2004) 239.

28 M. I. Jones, I. R. McColl, D. M. Grant, K. G. Parker, and T. L. Parker: J. Biomed. Mater. Res. 52 (2000) 413.

29 W. H. Park, W. S. Ha, H. Ito, T. Miyamoto, H. Inagaki, and Y. Noishiki: Fiber. Polym. 2 (2001) 58.

30 A. Vesel, M. Mozetic, and A. Zalar: Surf. Interface Anal. 40 (2008) 661.

31 D. J. Wilson, R. L. Williams, and R. C. Pond: Surf. Interface Anal. 31 (2001) 385.

32 G. C. Basak, A. Bandyopadhyay, S. Neogi, and A. K. Bhowmick: Appl. Surf. Sci. 257 (2011) 2891. 